\title{
Nonlinear dynamics of a quantum-dot laser coupled to an electro-optic modulator
}

\author{
Miriam Wegert, Daniel Schwochert, Eckehard Schöll, and Kathy Lüdge \\ Institut für Theoretische Physik, Technische Universität Berlin, D-10623 Berlin, Germany \\ Email: wegert@itp.tu-berlin.de
}

\begin{abstract}
We investigate the nonlinear dynamics of a quantum-dot laser coupled to an electro-optic modulator using the Lang-Kobayashi model. We determine the effect of the detuning between the EOM and the QD laser and the influence of the static phase shift on the QD laser dynamics. The electrooptical modulator's model is based on semiconductor MaxwellBloch equations with voltage dependent loss rates.
\end{abstract}

\section{INTRODUCTION}

Self-organized quantum dot (QD) laser structures with integrated electro-optic modulators (EOM) are attractive for applications in optical communication systems since electrooptic effects like the quantum-confined Stark effect (QCSE) are particularly strong in QDs. In this work we focus on the dynamics of an integrated device consisting of an EOM adjacent to a QD laser an investigate the influence of the EOM on the laser dynamics using a Lang-Kobayashi model [1] with an instantaneous feedback term due to the small length of the EOM.

\section{MODEL}
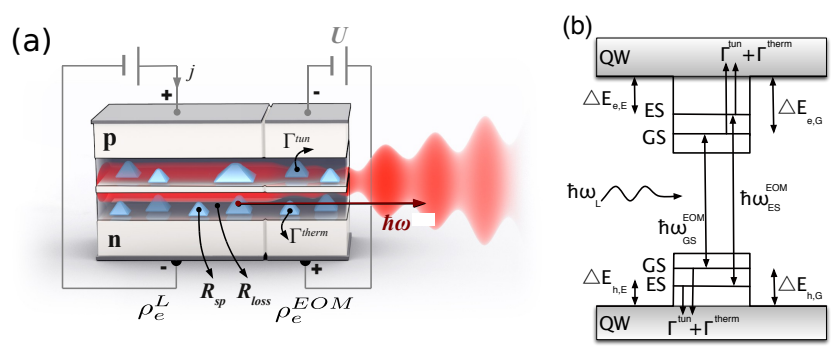

Fig. 1. (a) Scheme of the QD laser with integrated EOM. QDs (light blue pyramids) are surrounded by a QW. (b) Energy diagram of the QD-QW system inside the EOM structure.

Fig. 1(a) shows the scheme of the QD laser with integrated EOM. The QD laser's output is injected into the EOM and modulated due to the applied voltage $U$. Our EOM model is based on the QD semiconductor optical amplifier model described in [2], [3] but with different carrier capture and escape rates since the EOM is modulated by a reverse voltage. The EOM's model consists of optical Bloch equations for the quantum dot interband polarizations and electron- and hole occupations. The dynamical equations describing the dynamics inside the EOM consist of the QD interband polarizations $p_{m}$, describing the probability of an optical transition between the respective electron and hole levels and the QD carrier occupation probabilities $\rho_{e / h, m}^{E O M}$ of electrons $(e)$ and holes $(h)$ and are given by the following equations

$$
\begin{aligned}
\frac{\partial p_{m}}{\partial t}= & -i\left(\Delta \omega_{m}^{0}+\Delta \omega^{Q C S}(U)\right) p_{m} \\
& +\frac{\Omega(U)}{2 i}\left(\rho_{e, m}^{E O M}+\rho_{h, m}^{E O M}-1\right)-\frac{p_{m}}{T_{2}} \\
\frac{\partial \rho_{e / h, G S}^{E O M}}{\partial t}= & -\operatorname{Im}\left[\Omega(U) p_{G S}{ }^{*}\right]+2 R_{e / h}^{c a p}-2 R_{e / h}^{e s c} \\
& -\left(\Gamma_{e / h, G S}^{t u n}+\Gamma_{e / h, G S}^{t h e r m}\right) \rho_{e / h, G S}^{E O M}-R_{s p, G S} \\
\frac{\partial \rho_{e / h, E S}^{E O M}}{\partial t}= & -\operatorname{Im}\left[\Omega(U) p_{E S}{ }^{*}\right]-R_{e / h}^{c a p}+R_{e / h}^{e s c} \\
& -\left(\Gamma_{e / h, E S}^{t u n}+\Gamma_{e / h, E S}^{t h e r m}\right) \rho_{e / h, E S}^{E O M}-R_{s p, E S}
\end{aligned}
$$

The QD ground state (GS) and the first excited state (ES) inside the EOM are discriminated by a level index $m$. Scattering induced coherence loss for the QDs is accounted for by a dephasing time $T_{2}$. The EOM is modulated by a reverse voltage $U$. Due to the QCSE a red shift of the transition energy and a decrease of the oscillator strength occur. $\Delta \omega_{m}^{0}=\omega_{m}^{E O M}-\omega_{L}$ is the detuning between the unbiased EOM transition $\omega_{m}^{E O M}$ and the injected light field $\omega_{L} . \Delta \omega^{Q C S}(U)$ denotes the additional frequency detuning due to the QCSE as a function of the applied voltage $U$. The Rabi frequency of the QD transitions with voltage dependent dipole moment $\mu(U)$ enters as $\Omega(U)=\frac{\mu(U)}{\hbar} E$. Carrier transitions between the QD's GS and ES are given by $R_{e, h}^{c a p}$ and $R_{e, h}^{e s c}$. $\Gamma_{e / h}^{t u n}$ and $\Gamma_{e / h}^{\text {therm }}$ are voltage dependent loss rates into the quantum well (QW) due to tunneling and thermal excitation, respectively. Losses due to spontaneous emission in the QDs are given by $R_{s p, m}$. Fig. 1(b) shows the bandstructure of the EOM material. The QD GS energy levels are adjusted to $\Delta E_{e, G}=210 \mathrm{meV}$ below the QW band edge for electrons and $\Delta E_{h, G}=50 \mathrm{meV}$ for holes. The ES electron energy levels are $\Delta E_{e, E}=146 \mathrm{meV}$ and hole levels $\Delta E_{h, E}=44 \mathrm{meV}$ below the band edge, respectively. The complex slowly varying electric field amplitude $E(t)$ is injected from the QD laser into the EOM. Absorption and phase shift within the EOM are given as the real and imaginary part of the complex amplitude 
gain [4]

$$
\begin{aligned}
g\left(\omega_{L}, t\right)= & \frac{i \omega_{L} \Gamma}{\epsilon_{b g} \epsilon_{0}} \frac{N^{Q D}}{h^{Q W}} \mu^{*} \frac{-\mu T_{2}}{2 \hbar} \\
& {\left[\sum_{m}\left(\rho_{e, m}^{E O M}+\rho_{h, m}^{E O M}-1\right) \frac{\Delta \omega_{m}(U) T_{2}+i}{1+\left(\Delta \omega_{m}(U) T_{2}\right)^{2}}\right] . }
\end{aligned}
$$

$\Gamma$ is the geometric confinement factor, $\epsilon_{b g}$ the background permittivity, $h^{Q W}$ the height of each QW layer and $N^{Q D}$ the QD areal density per QW layer. $\Delta \omega_{m}(U)=\Delta \omega_{m}^{0}+\Delta \omega^{Q C S}(U)$ denotes the total frequency detuning between the laser and the EOM. Details can be found in [4]. The rate equations modelling the electron and hole occupation probabilities in the QDs, $\rho_{e}^{L}$ and $\rho_{h}^{L}$, and the electron and hole densities in the QW can be found in [5], [6]. The electric field inside the QD laser is given as

$$
\begin{array}{r}
\frac{\partial E}{\partial t}=\frac{1+i \alpha}{2}\left[2 \bar{W} Z_{a}^{Q D}\left(\rho_{e}^{L}+\rho_{h}^{L}-1\right)-\kappa\right] E+R_{s p} \\
+\frac{R}{\tau_{i n}} K(U, E) \exp \left[i\left(C(U, E)+C_{0}\right)\right] E
\end{array}
$$

where $R=\sqrt{\left(1-R_{\text {in }}\right) R_{\text {out }}}$ with $R_{\text {in }}$ and $R_{\text {out }}$ being the reflection coefficients between the QD laser and the EOM and between the EOM and air, respectively. $\alpha$ is the linewidth enhancement factor, $Z_{a}^{Q D}$ the total number of active QDs in the QW, $\bar{W}$ the Einstein coefficient for coherent interaction, $\kappa$ the internal loss rate and $\tau_{i n}$ the internal round-trip time. The feedback strength $K(U, E)$ and the phase shift $C(U, E)$ are calculated by Lambert-Beer's law using Eq. (4):

$$
\begin{array}{r}
K=\exp (2 \tau \cdot \operatorname{Re}(-g)) \\
C=2 \tau \cdot \operatorname{Im}(g)
\end{array}
$$

$C_{0}$ is a static phase shift given by the length of the EOM and its refractive index. Since the delay time $\tau$ lies in the time scale of the modulator dynamics, we checked whether the time delay could be neglected. Our calculations show that it is reasonable to use the instanteneous feedback term described above in this limit.

\section{COUPled QD LASER DYNAMics}

Using the set of equations described above the dynamics of a QD laser coupled to a modulated EOM is simulated numerically. For this purpose the EOM operates with a digital signal in a range of $-8 \mathrm{~V} \leq U(t) \leq-4 \mathrm{~V}$ with a frequency of $\nu=10 \mathrm{GHz}$. Since we want to explore the influence of the detuning on the QD laser and thus allow for an additional detuning $\Delta \omega_{m}^{0}$ between laser and unbiased EOM which does not change with the voltage. Fig. 2(a) shows the turn-on dynamics of the QD laser with feedback due to the EOM. The electric field strength of the QD laser does not reach a stationary value but oscillates with a small amplitude due to the periodic modulation of the EOM. Fig. 2(b) shows the minimum and maximum of these oscillations as a function of the static phase shift $C_{0}$ and for different values of the detuning $\Delta \omega_{G S}^{0}$. While the EOM operation is very sensitive to changes of the detuning, the QD laser output shows only small changes. The varying values of the electric field strength are not related to changes in the absorption inside the EOM but rather given by the phase shift $C$ which is sensitive to variations of the detuning. Fig. 2 shows that the modulation of the EOM transfers to the QD laser, albeit in a rather small variation compared to the steady state value of the electric field. The amplitude of the variation of the QD laser output can be controlled by the static phase shift $C_{0}$.
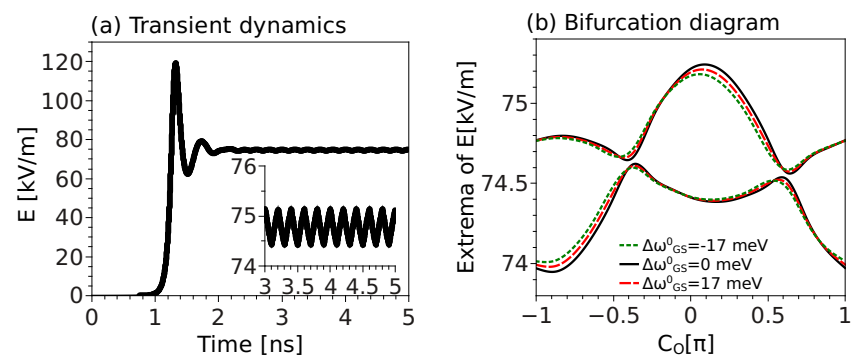

Fig. 2. (a) Turn-on dynamics of the QD laser, the inset shows the oscillations around the stationary value. (b) The plot shows minimum and maximum of the QD laser field strength depending on the static phase shift $C_{0}$ for three different values of the detuning $\Delta \omega_{G S}^{0}$. The voltage applied to the EOM changes between $U_{1}=-4 \mathrm{~V}$ and $U_{2}=-8 \mathrm{~V}$. Parameters: $\nu=10 \mathrm{GHz}$, $T_{2}=100 \mathrm{fs}, \alpha=0.9$ and $j=2 j_{t h}$.

\section{CONCLUSION}

Using a Maxwell-Bloch approach and the Lang-Kobayashi model with instantenous feedback we investigated the coupled dynamics of a QD laser and an EOM. While the QD laser is sensitive to changes of the static phase shift $C_{0}$, the detuning between the QD laser and the EOM influences the QD laser dynamics only slightly. Since a possible application are optical communication systems, it is important to be able to control the transient laser dynamics.

\section{ACKNOWLEDGMENT}

This work was supported by DFG in the framework of Sfb787.

\section{REFERENCES}

[1] R. Lang and K. Kobayashi, "External optical feedback effects on semiconductor injection laser properties," IEEE J. Quantum Electron., vol. 16, pp. 347-355, 1980.

[2] N. Majer, K. Lüdge, and E. Schöll, "Cascading enables ultrafast gain recovery dynamics of quantum dot semiconductor optical amplifiers," Phys. Rev. B, vol. 82, p. 235301, 2010.

[3] M. Wegert, N. Majer, K. Lüdge, S. Dommers-Völkel, J. Gomis-Bresco, A. Knorr, U. Woggon, and E. Schöll, "Nonlinear gain dynamics of quantum dot optical amplifiers," Semicond. Sci. Technol., vol. 26, p. $014008,2011$.

[4] B. Lingnau, K. Lüdge, W. W. Chow, and E. Schöll, "Failure of the $\alpha$ factor in describing dynamical instabilities and chaos in quantum-dot lasers," Phys. Rev. E, vol. 86, no. 6, p. 065201(R), 2012.

[5] C. Otto, B. Globisch, K. Lüdge, E. Schöll, and T. Erneux, "Complex dynamics of semiconductor quantum dot lasers subject to delayed optical feedback," Int. J. Bif. Chaos, vol. 22, no. 10, p. 1250246, 2012.

[6] J. Pausch, C. Otto, E. Tylaite, N. Majer, E. Schöll, and K. Lüdge, "Optically injected quantum dot lasers - impact of nonlinear carrier lifetimes on frequency locking dynamics," New J. Phys., vol. 14, p. 053018, 2012. 